\title{
Prehospital tranexamic acid: READY for primetime?
}

\author{
Joe Nemeth $^{1}$ (D) Alun Ackery ${ }^{2} \cdot$ François de Champlain $^{3}$
}

Received: 11 January 2021 / Accepted: 20 January 2021

(C) The Author(s), under exclusive licence to Canadian Association of Emergency Physicians (CAEP)/ Association Canadienne de Médecine d'Urgence (ACMU) 2021

Tranexamic acid (TXA) is an antifibrinolytic agent exerting its action on the coagulation process by competitively inhibiting plasminogen activation and ultimately reducing hemorrhage. Recent literature has provided strong support for the early use of TXA for severely injured patients. Its prominence as a treatment modality in trauma patients was initially highlighted in the Clinical Randomization of an Antifibrinolytic in Significant Hemorrhage (CRASH-2) study showing an all-cause mortality benefit in patients with known or suspected significant hemorrhage. However, questions persist regarding its optimal trauma-related indications and timing of administration.

In this issue of CJEM, Wong and colleagues characterize the clinical utilization of TXA by paramedics in British Columbia (BC) for acute major trauma [1]. This quality assurance retrospective chart review used a convenient sample of 100 patients, applying previously described CRASH-2 hemodynamical instability criteria (systolic blood pressure under $90 \mathrm{mmHg}$ or a heart rate over 110 beats per minute) to assess eligibility to receive prehospital administration of TXA. Descriptive statistics were adopted to report a 35\% utilization rate of TXA for eligible patients compared to $19 \%$ as previously reported for patients brought to a level 1 trauma center in BC [2]. This study was not designed to assess any

Joe Nemeth

Joe.nemeth@mcgill.ca

Alun Ackery

alun.ackery@unityhealth.to

François de Champlain

francois.de_champlain@mcgill.ca

1 Department of Emergency Medicine, Department of Pediatrics, McGill University, McGill University Health Center, Montréal, QC, Canada

2 Division of Emergency Medicine, Department of Medicine, University of Toronto, St- Michael's Hospital, Toronto, ON, Canada

3 Department of Emergency Medicine, McGill University, McGill University Health Center, Montréal, QC, Canada morbidity or mortality benefit of prehospital use of TXA. The average additional delay to reach hospital was reported to be 14 min added to an already long transport time. This is worrisome for patients suffering major trauma; however, commentary on this difference is problematic with the small sample size provided. Additionally, no hypothesis regarding any potential benefit of survival of patients receiving prehospital TXA can be generated from this study. Most importantly, this article highlights an important trauma topic: the role of TXA in the prehospital setting.

The concept of giving TXA in the prehospital setting is not a novel one. The more significant question remains whether prehospital administration of TXA adds any benefit not outweighed by potential harm, such as additional time spent on scene for a time-sensitive condition such as the management of a major trauma. Important considerations before widespread implementation of prehospital TXA would be to define: in which type of setting (urban or rural, proximity to a trauma center) and in which specific level of hemorrhagic compromise (based on physiologic/anatomic parameters) might a patient benefit from its administration, which level medic should be administering it (primary care or advanced/critical care), and the preferred modality of administration (1 g IV bolus followed by a $1 \mathrm{~g}$ perfusion versus $2 \mathrm{~g}$ IV bolus). Some evidence has emerged recently to potentially answer these questions.

A secondary analysis of the CRASH-2 trial conducted on 20,211 patients found that early treatment ( $\leq 1 \mathrm{~h}$ from injury) significantly reduced the risk of death to a greater extent (RR $0.6895 \%$ CI $0.57-0.82 ; p<0.0001$ ) compared to the group that received TXA between 1 and $3 \mathrm{~h}$ after injury (RR 0.79, 0.64-0.97; $p=0.03$ ) [3]. These findings supported the argument for earlier treatment and led many EMS to extrapolate these findings to prehospital settings.

The CRASH-3 trial, a multicenter randomized placebo control trial that looked at the use of TXA on 12,737 patients who suffered traumatic brain injury, did not find an overall survival benefit at 28 days for their primary endpoint but only for their pre-specified sensitivity analysis for patients 
presenting with a GCS score of 4-12 with some degree of pupillary reaction [4]. This study also found that earlier treatment was more effective for the subgroup of mild and moderate head trauma (GCS 9-15), further supporting the argument for use in the prehospital setting. It is clear that patients with very severe traumatic brain injury seem to have less to gain from the administration of TXA, which is all somewhat intuitive.

A recently published multicenter 3-arm randomized control trial evaluated the impact of prehospital TXA given to patients suffering from moderate or severe traumatic brain injury on the 6-month neurologic outcomes defined by the Glasgow Outcome Scale-Extended (GOSE) score [5]. In this study, TXA had to be given earlier (within $2 \mathrm{~h}$ of injury) but did not find any statistical difference on the 6-month favorable neurologic outcome (GOSE score $>4$ ), on 28-day mortality or on any other secondary endpoints. There was also a higher incidence of seizures, a known adverse effect of TXA, in the group that received $2 \mathrm{~g}$ IV bolus versus $1 \mathrm{~g}$ IV bolus followed by $1 \mathrm{~g} 8$-h infusion ( $5 \%$ versus $2 \%$ ) as well as higher rates of thromboembolic event (9\% vs $4 \%$ ). Guyette et al., however, recently showed that higher upfront dosing did not result in more adverse effects [6]. These findings, even though not conclusive, should nevertheless heed caution regarding use of the $2 \mathrm{~g}$ IV bolus regimen until further dosage trials are conducted. Another important finding is that only $56 \%$ of 966 patients enrolled in this study had intracranial hemorrhage, highlighting the difficulty in making this diagnosis only from the GCS score in the prehospital setting. Also, 20\% of patients had a higher GCS score than enrolment criteria at hospital, illustrating the difficulty of accurate assessment of the prehospital GCS score. This is a challenge of out-of-hospital research in general where enrolment is not based on a firm diagnosis. Giving TXA to patients who do not have traumatic brain injury will greatly increase the number needed to treat and may dilute any potential prehospital benefit for use.

We firmly believe that administration of prehospital TXA should not delay transfer of a significantly injured patient to the nearest appropriate hospital by regional EMS protocols.
Therefore, it might be more suitable to administer TXA en route or during transportations expected to be longer than in most urban settings under normal conditions.

In summary, there is still a paucity of evidence supporting universal prehospital use of TXA in the major trauma patient as well as a lack of consensus in the selection of patients with traumatic brain injury that might benefit from it. Therefore, more studies designed to address these specific questions are needed before widespread prehospital implementation is ready for primetime, but it is unlikely that better-powered studies on TXA than CRASH-2 and CRASH-3 will be conducted in the future.

\section{Compliance with ethical standards}

Conflict of interest The authors declare that they have no conflict of interest.

\section{References}

1. Wong et al. Assessing the Clinical Utilization of TranExamic acid by paramedics for patients with major trauma (ACUTE)

2. $\mathrm{Ng} \mathrm{M}$, et al. Evaluation of tranexamic acid in trauma patients: a retrospective quantitative analysis. Am J Emerg Med. 2019;37(3):444-9.

3. CRASH-2 collaborators. The importance of early treatment with tranexamic acid in bleeding trauma patients: an exploratory analysis of the CRASH-2 randomised controlled trial. Lancet. 2011; 377: 1096-101.

4. CRASH-3 trial collaborators. Effects of tranexamic acid on death, disability, vascular occlusive events and other morbidities in patients with acute traumatic brain injury (CRASH-3): a randomised, placebo-controlled trial. Lancet. 2019;394(10210):1713-1723.

5. Rowell SE, et al. Effect of out-of-hospital tranexamic acid vs placebo on 6-month functional neurologic outcomes in patients with moderate or severe traumatic brain injury. JAMA. 2020;324(10):961-74.

6. Guyette FX, et al. Tranexamic acid during prehospital transport in patients at risk for hemorrhage after injurya double-blind, placebo-controlled. Randomized Clinical Trial JAMA Surg. 2020. https://doi.org/10.1001/jamasurg.2020.4350. 